\title{
Quasi-linear dynamics of Weibel instability
}

\author{
O. A. Pokhotelov ${ }^{1}$ and O. A. Amariutei ${ }^{2}$ \\ ${ }^{1}$ Automatic Control and Systems Engineering, University of Sheffield, Sheffield, UK \\ ${ }^{2}$ Finnish Meteorological Institute, Geophysical Research Division P.O. Box 50300101 Helsinki, Finland
}

Received: 5 September 2011 - Revised: 28 October 2011 - Accepted: 28 October 2011 - Published: 4 November 2011

\begin{abstract}
The quasi-linear dynamics of resonant Weibel mode is discussed. It is found that nonlinear saturation of Weibel mode is accompanied by substantial modification of the distribution function in resonant region. With the growth of the wave amplitude the parabolic bell-like form of the electron distribution function in this region converts into flatter shape, such as parabola of the fourth order. This results in significant weakening of the resonant interaction of the wave with particles. The latter becomes weaker and then becomes adiabatic interaction with the bulk of the plasma. This is similar to the case of Bernstein-Greene-Kruskal (Bernstein et al., 1957) electrostatic waves. The mathematical similarity of the Weibel and magnetic mirror instabilities is discussed.
\end{abstract}

Keywords. Interplanetary physics (Plasma waves and turbulence)

\section{Introduction}

In 1959 E. Weibel showed (Weibel, 1959) that velocity space anisotropy instability includes a growing purely aperiodic electromagnetic mode even in the absence of an external magnetic field. This instability generates a magnetic field in a finite range of wave numbers. The ions in this process form a fixed neutralizing background. Far from the instability threshold when the temperature anisotropy is very strong the Weibel instability transforms into the filamentation instability.

Weibel instability is quite common in laboratory and astrophysical plasmas. It serves as one of the basic mechanisms of conversion of the free energy stored in the inversely populated plasma into magnetic field energy. It can be driven in pulsar winds (Kazimura et al., 1998), gamma-ray bursts, and in their afterglows (Medvedev and Loeb, 1999; Gedalin

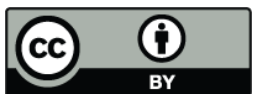

Correspondence to: O. A. Pokhotelov (o.a.pokhotelov@sheffield.ac.uk) et al., 2010). Recently Schlickeiser and Shukla (2003) discussed the generation of cosmological magnetic fields by the Weibel instability. In terms of the mathematical description the Weibel instability is somewhat similar to the magnetic mirror instability (Pokhotelov et al., 2010). Furthermore, the Weibel-type instabilities can play an important role in the plasma processes related to magnetic reconnection occurring in the vicinity of the Earth's magnetopause and in the magnetic tail (Karimabadi et al., 2004; Ricci et al., 2004; Daughton et al., 2004).

The paper is structured as follows: in Sect. 2 we derive the Weibel dispersion relation for the arbitrary form of the electron distribution function. Section 3 is devoted to the study of quasi-linear modification of the electron distribution function. It is shown that major modification occurs in the resonant region. The derivation of the nonlinear instability growth rate and estimation of the amplitude level in the saturated state is given in Sect. 4. Our discussion and conclusions are found in Sect. 5 .

\section{Basic equations}

We consider the electron-ion plasma where ions have the standard Maxwellian distribution and electrons take the anisotropic bi-Maxwellian form

$F_{0}=\frac{n}{\pi v_{T_{\mathrm{x}}} v_{T_{\mathrm{Z}}}} \exp \left(-\frac{v_{\mathrm{x}}^{2}}{v_{T_{\mathrm{x}}}^{2}}-\frac{v_{\mathrm{z}}^{2}}{v_{T_{\mathrm{z}}}^{2}}\right)$,

where $F_{0}$ is the electron velocity distribution function integrated over $v_{\mathrm{y}}, n$ is the plasma number density, $v_{T_{\mathrm{x}(\mathrm{z})}}=$ $\left(2 T_{\mathrm{x}(\mathrm{z})} / m\right)^{1 / 2}$ is the $\mathrm{x}-(\mathrm{z}-)$ component of the thermal electron velocity, $T_{\mathrm{x}(\mathrm{z})}$ is the $\mathrm{x}-(\mathrm{z}-)$ component of the electron temperature and $m$ is the electron mass. For non-Maxwellian distribution functions the Weibel instability has been recently analyzed by Zaheer and Murtaza (2007). The wave phase velocity of the Weibel mode is much greater than the ion

Published by Copernicus Publications on behalf of the European Geosciences Union. 
thermal velocity and due to that the ions do not contribute to the Weibel mode dispersion relation.

The magnetic and electric fields are chosen to be linearly polarized and normal to the wave vector. The Fourier components of the electric and magnetic fields $\boldsymbol{E}$ and $\boldsymbol{B}$ are connected through the impedance ratio (Faradey's law)

$E_{\mathrm{x}} / B_{\mathrm{y}}=\omega / k \quad$ with $\quad B_{\mathrm{y}}=B_{k} \exp (-i \omega t+i k z)$,

where $\omega$ and $k$ are the wave frequency and the wave number, respectively.

The pertubation of the electron distribution function for the Weibel-type perturbations is

$\delta F_{\mathbf{k}}=\frac{i e}{m} \frac{B_{\mathbf{k}}}{k} v_{\mathrm{x}}\left(\frac{\partial F}{v_{\mathrm{x}} \partial v_{\mathrm{x}}}-\frac{\partial F}{v_{\mathrm{z}} \partial v_{\mathrm{z}}}+\frac{\omega}{\omega-k v_{\mathrm{z}}} \frac{\partial F}{v_{\mathrm{Z}} \partial v_{\mathrm{Z}}}\right)$,

where $F$ is the background electron distribution function.

The first two terms on the r.h.s. of Eq. (3) in the round brackets describe the effect of the electron temperature anisotropy. Recalling the case of the magnetic mirror instability (Pokhotelov et al., 2002) one finds that they are similar to the action of the mirror force. The last term is due to the interaction with resonant electrons.

We consider the low-frequency limit $\omega \ll k v_{z}$. In this case the third term in Eq. (3) is always small except for the particles having very small $v_{z}$ velocities. We note that contribution of these particles can be comparable or even greater than that coming from the hydromagnetic terms. The expansion of the resonant denominator in this case reads $\left(\omega-k v_{\mathrm{Z}}\right)^{-1}=$ $-i \pi \delta\left(v_{\mathrm{z}}\right) /|k|-P\left(1 / k v_{\mathrm{z}}+\omega / k^{2} v_{\mathrm{z}}^{2}\right)$, where $\delta(x)$ is the Dirac delta function and $P$ stands for the Cauchy principal value. With the help of this expansion the perturbation of the distribution function takes the form

$$
\begin{aligned}
\delta F_{\mathbf{k}}= & \frac{i e}{m} \frac{B_{\mathbf{k}}}{k} v_{\mathrm{x}}\left[\frac{\partial F}{v_{\mathrm{x}} \partial v_{\mathrm{x}}}-\frac{\partial F}{v_{\mathrm{z}} \partial v_{\mathrm{z}}}\right. \\
& \left.-\frac{i \pi \omega}{|k|} \delta\left(v_{\mathrm{z}}\right) \frac{\partial F}{v_{\mathrm{z}} \partial v_{\mathrm{z}}}-P\left(\frac{\omega}{k} \frac{\partial F}{v_{\mathrm{z}}^{2} \partial v_{\mathrm{z}}}+\frac{\omega^{2}}{k^{2}} \frac{\partial F}{v_{\mathrm{z}}^{3} \partial v_{\mathrm{z}}}\right)\right]
\end{aligned}
$$

Making use of Eq. (4) and the Ampére's law one obtains the Weibel dispersion relation for the arbitrary form of the unperturbed distribution function

$$
\begin{gathered}
I_{2}-1-\frac{k^{2} c^{2}}{\omega_{p}^{2}}-\frac{i \pi \omega}{|k| n} \int v_{\mathrm{x}}^{2} \frac{\partial F}{v_{\mathrm{z}} \partial v_{\mathrm{z}}} \delta\left(v_{\mathrm{z}}\right) d v_{\mathrm{x}} d v_{\mathrm{z}} \\
+\frac{\omega^{2}}{k^{2}} I_{1}=0
\end{gathered}
$$

where $c$ is the velocity of light and $\omega_{p}$ is the Langmuir frequency.

We took into account that contribution of the term containing $P\left(1 / k v_{\mathrm{z}}\right)$ in the expansion of the resonant denominator vanishes and the expressions for $I_{1}$ and $I_{2}$ are given by

$$
I_{1}=-n^{-1} P\left(\int v_{\mathrm{x}}^{2} \frac{\partial F}{v_{z}^{3} \partial v_{z}} d v_{\mathrm{x}} d v_{\mathrm{z}}\right)
$$

and

$I_{2}=-n^{-1} \int v_{\mathrm{x}}^{2} \frac{\partial F}{v_{\mathrm{z}} \partial v_{\mathrm{z}}} d v_{\mathrm{x}} d v_{\mathrm{z}}$

In contrast to the standard dispersion equation for the zerofrequency dispersion relation Eq. (5) is quadratic equation. Of course the addition term, containing $\omega^{2}$, is small as $\omega^{2} / k^{2} v_{\mathrm{T}}^{2} \ll 1$ (where $v_{\mathrm{T}}$ is the electron characteristic thermal velocity) relative to other terms. However, as it will be shown in what follows, in the nonlinear regime it may play an important role.

In the linear limit decomposing the wave frequency in the dispersion relation (5) one obtains

$$
\begin{gathered}
\omega_{R}\left(\frac{\pi}{|k| n} \int v_{\mathrm{x}}^{2} \frac{\partial F}{v_{\mathrm{z}} \partial v_{\mathrm{z}}} \delta\left(v_{\mathrm{z}}\right) d v_{\mathrm{x}} d v_{\mathrm{z}}-\frac{2 \gamma}{k^{2}} I_{1}\right) \\
\simeq \omega_{R} \frac{\pi}{|k| n} \int v_{\mathrm{x}}^{2} \frac{\partial F}{v_{\mathrm{z}} \partial v_{\mathrm{z}}} \delta\left(v_{\mathrm{z}}\right) d v_{\mathrm{x}} d v_{\mathrm{z}}=0,
\end{gathered}
$$

which shows that Weibel mode is nonpropagating zerofrequency mode $\omega_{R}=0$. It becomes growing when $I_{2}-1>$ 0 with the growth rate given by

$\gamma=-\frac{|k| n}{\pi} \frac{\left(I_{2}-1-\frac{k^{2} c^{2}}{\omega_{p}^{2}}\right)}{\int v_{\mathrm{x}}^{2} \frac{\partial F}{v_{\mathrm{z}} \partial v_{\mathrm{z}}} \delta\left(v_{\mathrm{z}}\right) d v_{\mathrm{x}} d v_{\mathrm{z}}}$.

The maximum growth rate is attained at $k=k_{\max }$ given by

$k_{\max }=\frac{\omega_{p}}{3^{1 / 2} c}\left(I_{2}-1\right)^{1 / 2}$,

and is

$\gamma_{\max }=\frac{2 n}{3^{3 / 2} \pi} \frac{\omega_{p}}{c} \frac{\left(I_{2}-1\right)^{3 / 2}}{\int v_{\mathrm{x}}^{2}\left(\partial F / v_{\mathrm{z}} \partial v_{\mathrm{z}}\right) \delta\left(v_{\mathrm{z}}\right) d v_{\mathrm{x}} d v_{\mathrm{z}}}$.

Substituting into Eq. (11) the bi-Maxwellian velocity distribution (1) one finds

$\gamma=\frac{|k| v_{T_{\mathrm{z}}}}{\pi^{1 / 2}} \frac{T_{\mathrm{z}}}{T_{\mathrm{X}}}\left(\frac{T_{\mathrm{x}}}{T_{\mathrm{Z}}}-1-\frac{c^{2} k^{2}}{\omega_{p}^{2}}\right)$.

The expressions for the optimal value of the wave number $k=k_{\max }$ and maximum value of the growth rate in the case of bi-Maxwellian distribution are now take the form

$k_{\max }=\frac{\omega_{p}}{3^{1 / 2} c}\left(\frac{T_{\mathrm{x}}}{T_{\mathrm{z}}}-1\right)^{1 / 2}$,

and

$\gamma_{\max }=\frac{2}{3^{3 / 2}} \omega_{p} \frac{v_{T_{\mathrm{z}}}}{c}\left(\frac{T_{\mathrm{x}}}{T_{\mathrm{z}}}-1\right)^{3 / 2}$.

Actually, the quantity $I_{2}-1$ defines the instability threshold for the arbitrary distribution function. In the particular case of bi-Maxwellian electrons it reduces to the standard value of threshold condition, $T_{\mathrm{x}} / T_{\mathrm{Z}}-1 \ll 1$. 


\section{Nonlinear variation of the electron velocity distribu- tion function}

In order to incorporate the effect of quasi-linear (QL) modification of the distribution function we assume that the coefficients in Eq. (3) are not fixed but slowly vary under the action of the QL effects. The equation that controls the QL modification can easily be obtained by making the space averaging of Vlasov equation and is

$\frac{\partial F}{\partial t}=\frac{e}{m} \sum_{\mathbf{k}}\left(\boldsymbol{E}_{\mathbf{k}}^{*}+\mathbf{v} \times \boldsymbol{B}_{\mathbf{k}}^{*}\right) \frac{\partial \delta F_{\mathbf{k}}}{\partial \mathbf{v}}$,

where asterix denotes the complex conjugate.

Substituting Eq. (3) into Eq. (15) one finds

$$
\frac{\partial F}{\partial t}=\frac{e^{2}}{m^{2}} v_{x}^{2} \sum_{\mathbf{k}} \gamma_{\mathbf{k}} \frac{\left|B_{\mathbf{k}}\right|^{2}}{k^{2}} \frac{\partial}{\partial v_{\mathrm{z}}}\left(\frac{1}{v_{\mathrm{Z}}^{2}} \frac{\partial F}{\partial v_{\mathrm{z}}}\right) .
$$

We took into account that Weibel instability is the zerofrequency mode and thus the frequency $\omega_{\mathbf{k}}$, entering Eq. (3), can be replaced by $i \gamma_{\mathbf{k}}$, where $\gamma_{\mathbf{k}}$ the wave amplitude growth rate. Furthermore, the major change in the distribution function occurs in the region of small $v_{\mathrm{z}}$ velocities. This allows us in the right-hand side of Eq. (16) to retain only the strongest term responsible for the parallel (along $v_{\mathrm{Z}}$ ) quasi-linear diffusion.

Making use of the relation

$\frac{\partial\left|B_{\mathbf{k}}\right|^{2}}{\partial t}=2 \gamma_{\mathbf{k}}\left|B_{\mathbf{k}}\right|^{2}$

Eq. (16) reduces to

$\frac{\partial F}{\partial h}=\frac{v_{\mathrm{x}}^{2}}{2} \frac{\partial}{\partial v_{\mathrm{z}}}\left(\frac{1}{v_{\mathrm{z}}^{2}} \frac{\partial F}{\partial v_{\mathrm{z}}}\right)$,

where the quantity $h$ is given by

$h=\frac{e^{2}}{m^{2}} \sum_{\mathbf{k}} \frac{\left|B_{\mathbf{k}}\right|^{2}}{k^{2}}$.

We separate the variables $h$ and $v_{\mathrm{z}}$ writing $F$ as $F=$ $\Phi(h) \Psi\left(v_{\mathrm{Z}}\right)$. Then one obtains

$\frac{2 \partial \ln \Phi}{v_{\mathrm{x}}^{2} \partial h}=\frac{1}{\Psi} \frac{\partial}{\partial v_{\mathrm{z}}}\left(\frac{1}{v_{\mathrm{z}}^{2}} \frac{\partial \Psi}{\partial v_{\mathrm{z}}}\right)=-4 \lambda^{2}$,

where eigenvalue $\lambda$ is an unspecified parameter. From Eq. (20) one finds

$\Phi=\Phi_{0} e^{-2 \lambda^{2} v_{x}^{2} h}$,

and

$\frac{\partial}{\partial v_{\mathrm{Z}}}\left(\frac{1}{v_{\mathrm{Z}}^{2}} \frac{\partial \Psi}{\partial v_{\mathrm{Z}}}\right)+4 \lambda^{2} \Psi=0$.

Here Eq. (22) is written in the Sturm-Liouville form. The value of $\lambda$ is not specified in this equation. Finding the value of $\lambda$ for which there exists a non-trivial solution of Eq. (22) satisfying the boundary conditions is a part of the SturmLiouville problem.

Such values of $\lambda$ when they exist are called the eigenvalues of the boundary value problem defined by Eq. (22) and prescribed set of boundary conditions. The corresponding solutions (for such a $\lambda$ ) are the eigenfunctions of this problem and are

$\Psi_{\lambda}=\left|v_{\mathrm{Z}}\right|^{3 / 2}\left[C_{1} J_{-3 / 4}\left(\lambda v_{\mathrm{Z}}^{2}\right)+C_{2} J_{3 / 4}\left(\lambda v_{\mathrm{Z}}^{2}\right)\right]$.

The solution of Eq. (22) must be bounded at $v_{\mathrm{Z}}=0$. This assumes that $C_{2}=0$. Thus, the particular solution of Eq. (22) is

$F_{\lambda} \propto\left|v_{\mathrm{z}}\right|^{3 / 2} e^{-4 \lambda^{2} v_{\mathrm{x}}^{2} h_{e}} J_{-3 / 4}\left(\lambda v_{\mathrm{z}}^{2}\right)$.

The general solution of Eq. (18) can be written in the form of Fourier-Bessel integral

$$
\begin{aligned}
F\left(v_{\mathrm{x}}, v_{\mathrm{Z}}, h\right)= & A\left|v_{z}\right|^{3 / 2} \int_{0}^{\infty} e^{-2 \lambda^{2} v_{\mathrm{x}}^{2} h} \lambda J_{-3 / 4}\left(\lambda v_{\mathrm{Z}}^{2}\right) \\
& \times \int_{0}^{\infty} \xi^{1 / 4} J_{-3 / 4}(\lambda \xi) e^{-\frac{\xi}{v_{T_{\mathrm{Z}}}^{2}}} d \xi d \lambda,
\end{aligned}
$$

where

$A=\frac{n}{\pi v_{T_{\mathrm{x}}} v_{T_{\mathrm{Z}}}} \exp \left(-\frac{v_{\mathrm{x}}^{2}}{v_{T_{\mathrm{x}}}^{2}}\right)$.

With the help of the relation

$\int_{0}^{\infty} \lambda J_{-3 / 4}\left(\lambda v_{\mathrm{Z}}^{2}\right) J_{-3 / 4}(\lambda \xi) d \lambda=\frac{\delta\left(\xi-v_{\mathrm{Z}}^{2}\right)}{v_{\mathrm{z}}^{2}}$.

one easily verifies that the initial condition $F(h=0)=F_{0}$ is satisfied.

The internal integral can be easily calculated and we find

$$
\begin{gathered}
F\left(v_{\mathrm{x}}, v_{\mathrm{Z}}, h\right)=\frac{A 2^{1 / 4} \Gamma(3 / 4)}{\pi^{\frac{1}{2}} v_{T_{\mathrm{Z}}}^{2}}\left|v_{\mathrm{Z}}\right|^{3 / 2} \\
\int_{0}^{\infty} \frac{e^{-2 \lambda^{2} v_{\mathrm{x}}^{2} h} \lambda^{1 / 4} J_{-3 / 4}\left(\lambda v_{\mathrm{Z}}^{2}\right)}{\left(\lambda^{2}+v_{T_{\mathrm{Z}}}^{-4}\right)^{3 / 4}} d \lambda,
\end{gathered}
$$

where $\Gamma(x)$ is the Gamma function.

The plot of $F$ as the function of $v_{z}$ for different values of $h$ and a given value of $v_{\mathrm{x}}$ can be made in a similar way as for the case of magnetic mirror mode (see Fig. 1 from Pokhotelov et al., 2010). One sees that QL diffusion leads to substantial flattening of the distribution function at small $v_{\mathrm{Z}}$. Instead of initial dependence $\propto \exp \left(-v_{\mathrm{Z}}^{2} / v_{T_{\mathrm{Z}}}^{2}\right)$ the velocity distribution function now scales as $\propto \exp \left(-v_{\mathrm{Z}}^{4} / v_{T_{\mathrm{Z}}}^{4}\right)$ and thus the term containing the $\delta\left(v_{\mathrm{z}}\right)$ function as well as that containing $P\left(1 / v_{\mathrm{z}}\right)$ vanish and thus the next term in the expansion of the denominator $\left(\omega-k v_{\mathrm{Z}}\right)^{-1}$ start to play a major role. This term is responsible for the weaker adiabatic interaction of electrons with the Weibel mode. 


\section{Nonlinear growth rate}

Using expression (28) one can calculate the quantities $I_{1}$ and $I_{2}$.

$I_{1}=-n^{-1} P\left(\int v_{\mathrm{x}}^{2} \frac{\partial F}{v_{\mathrm{z}}^{3} \partial v_{\mathrm{z}}} d v_{\mathrm{x}} d v_{\mathrm{z}}\right)=\beta \frac{v_{T_{\mathrm{x}}}^{3 / 2}}{v_{T_{\mathrm{z}}}^{3} h^{1 / 4}}$,

where $\beta=-(3 / 32) \Gamma(-3 / 4) \Gamma(1 / 4) \simeq 1.6$.

Our small parameter now becomes $\omega / k \Delta v_{*}$, where $\Delta v_{*} \simeq$ $h^{1 / 4} v_{T_{\mathrm{X}}}^{1 / 2}$. This is connected with the flattening of the distribution function and occurrence of the "plateau" in the resonant region, i.e. when $v_{\mathrm{Z}}$ is small.

Furthermore, we find

$I_{2}=\frac{T_{\mathrm{x}}}{T_{\mathrm{z}}}-\alpha\left(\frac{T_{\mathrm{x}}}{T_{\mathrm{z}}}\right)^{5 / 4} \frac{h^{1 / 4}}{v_{T_{\mathrm{z}}}^{1 / 2}}$,

where

$\alpha=-\frac{3}{2} \frac{\Gamma(3 / 4) \Gamma(-1 / 4)}{\Gamma^{2}(1 / 4)} \simeq 0.68$.

Substituting expressions for $I_{1}$ and $I_{2}$ into dispersion relation (5) one finds the instability growth rate

$\gamma=\frac{|k| v_{T_{\mathrm{z}}}^{3 / 2} h^{1 / 8}}{\beta^{1 / 2} v_{T_{\mathrm{x}}}^{3 / 4}}\left(\frac{T_{\mathrm{x}}}{T_{\mathrm{z}}}-1-\frac{c^{2} k^{2}}{\omega_{p}^{2}}-\alpha\left(\frac{T_{\mathrm{x}}}{T_{\mathrm{z}}}\right)^{5 / 4} \frac{h^{1 / 4}}{v_{T_{\mathrm{z}}}^{1 / 2}}\right)^{1 / 2}$

For the most growing mode defined by Eq. (5) one obtains

$$
\begin{aligned}
\gamma_{\max }= & \frac{2|k| v_{T_{\mathrm{z}}}^{3 / 2} h^{1 / 8}}{3^{3 / 2} \beta^{1 / 2} v_{T_{\mathrm{x}}}^{3 / 4}}\left(\frac{T_{\mathrm{x}}}{T_{\mathrm{z}}}-1\right)^{1 / 2} \\
& \times\left(\frac{T_{\mathrm{x}}}{T_{\mathrm{z}}}-1-\frac{3}{2} \alpha\left(\frac{T_{\mathrm{x}}}{T_{\mathrm{z}}}\right)^{5 / 4} \frac{h^{1 / 4}}{v_{T_{\mathrm{z}}}^{1 / 2}}\right)^{1 / 2} .
\end{aligned}
$$

One sees that QL saturation of this mode is attained at relatively small amplitude when

$h=v_{T_{\mathrm{z}}}^{2}\left(\frac{3}{2}\right)^{4} \frac{1}{\alpha^{4}}\left(\frac{T_{\mathrm{z}}}{T_{\mathrm{x}}}\right)^{5}\left(\frac{T_{\mathrm{x}}}{T_{\mathrm{z}}}-1\right)^{4}$.

A further nonlinear evolution of the Weibel mode will be controlled by the effects that were neglected in the course of our analysis such as the mode coupling and nonlinear variations of the skin depth. Similar effects play an important role, for example, in the nonlinear evolution of mirror instability (Pokhotelov et al., 2008; Califano et al., 2008; Balikhin et al., 2009, 2010; Hellinger et al., 2009). However, detailed consideration of these effects is outside the scope of the present study.

\section{Discussion and conclusions}

The present paper describes QL stabilization of the Weibel instability associated with fast modification of the electron velocity distribution function in resonant region. In the course of the instability saturation the resonant term (the Landau term) is rapidly out of the game and its role is then passed to the adiabatic interaction of the mode with the bulk of the plasma. In terms of mathematical description this scenario has much in common with nonlinear evolution of magnetic mirror instability (Pokhotelov et al., 2002, 2010) which is controlled by similar physical processes. The corresponding nonlinear growth rate (Eq. 33) was calculated and estimation of the level of the turbulent pulsations has been made (Eq. 34). The description of further nonlinear evolution is controlled by wave-wave interactions (e.g. Brodin and Stenflo, 1989) and requires the use of numerical simulations. Some aspects of this nonlinear dynamics were presented by Palodhi et al. (2009).

Acknowledgements. This research was supported by STFC and by RFBR (grant No 11-05-00920).

Guest Editor M. Balikhin thanks two anonymous referees for their help in evaluating this paper.

\section{References}

Balikhin, M. A., Sagdeev, R. Z., Walker, S. N., Pokhotelov, O. A., Sibeck, D. G., Beloff, N., and Dudnikova, G.: THEMIS Observations of Mirror Structures: Magnetic Holes and Instability Thresholds, Geophys. Res. Lett., 36, LO3105, doi:10.1029/2008GL036923, 2009.

Balikhin, M., Pokhotelov, O., Walker, S., Boynton, R., and Beloff, N.: Mirror mode peaks: THEMIS observations versus theories, Geophys. Res. Lett., 37, L05104, doi:10.1029/2009GL042090, 2010.

Bernstein, I. B., Greene, J. M., and Kruskal, M. D.: Exact nonliner plasma oscillations, Phys. Rev., 108, 546-550, 1957.

Brodin, G. and Stenflo, L.: Three-wave interaction between transverse and longitudinal waves, J. Plasma Phys., 42, part2, 187, 1989.

Califano, F., Hellinger, P., Kuznetsov, E. A., Passot, T., Sulem, P. L., and Trávniček, P. M.: Nonlinear mirror mode dynamics: Simulations and modelling, J. Geophys. Res., 113, A08219, doi:10.1029/2007JA012898, 2008.

Daughton, W., Lapenta, G., and Ricci, P.: Nonlinear evolution of the lower-hybrid drift instability in current sheet, Phys. Rev. Lett., 93, 105004, doi:10.1103/PhysRevLett.93.105004, 2004.

Gedalin, M., Medvedev, M., Spitkovsky, A., Krasnoselskikh, V., Balikhin, M., Vaivads, A., and Perri, S.: Growth of filaments and saturation of the filamentation instability, Phys. Plasmas, 17, 032108, doi:10.1063/1.3345824, 2010.

Hellinger, P., Kuznetsov, E. A., Passot, T., Sulem, P. L., and Trávniček, P. M.: Mirror instability: From quasi-linear diffusion to coherent structures, Geophys. Res. Lett., 36, L06103, doi:10.1029/2008GL036805, 2009.

Karimabadi, H., Daughton, W., and Quest, K. B.: Geophys. Res. Lett., 31, L18801, doi:1029/2004/GLO020791, 2004. 
Kazimura, Y., Sakai, J. I., Neubert, T., and Bulanov, S. V.: Generation of a small-scale quasi-static magnetic field and fast particles during the collision of electron-positron plasma clouds, Astrophys. J. Lett., 498, L183, 1998.

Medvedev, M. V. and Loeb, A.: Generation of magnetic fields in the relativistic shock of gamma-ray burst sources, Astrophys. J., 526, 697-706, doi:10.1086/308038, 1999.

Palodhi, L., Califano, F., and Pegoraro, F.: Nonlinear kinetic development of the Weibel instability and the generation of electrostatic coherent structures, Plasma Phys. Control. Fusion, 51, 125006, doi:10.1088/0741-3335/51/12/125006, 2009.

Pokhotelov, O. A., Treumann, R. A., Sagdeev, R. Z., Balikhin, M. A., Onishchenko, O. G., Pavlenko, V. P., and Sandberg, I.: Linear theory of the mirror instability in non-Maxwellian space plasmas, J. Geophys. Res., 107, 1312, doi:10.1029/2001JA009125, 2002.

Pokhotelov, O. A., Sagdeev, R. Z., Balikhin, M. A., Onishchenko, O. G., and Fedun, V. N.: Nonlinear mirror waves in nonMaxwellian space plasmas, J. Geophys. Res., 113, A04225, doi:10.1029/2007JA012642, 2008.
Pokhotelov, O. A., Sagdeev, R. Z., Balikhin, M. A., Fedun, V. N., and Dudnikova, G. I.: Nonlinear Mirror and Weibel modes: peculiarities of quasi-linear dynamics, Ann. Geophys., 28, 21612167, doi:10.5194/angeo-28-2161-2010, 2010.

Ricci, P., Brackbill, J. U., Daughton, W., and Lapenta, G.: Influence of the lower hybrid drift instability in the onset of magnetic reconnection, Phys. Plasmas, 11, 4489-4500, doi:10.1063/1.1778744, 2004.

Schlickeiser, R. and Shukla, P. K.: Cosmological magnetic field generation by the Weibel instability, Astrophys. J. Lett., 599, L57, 2003.

Weibel, E. S.: Spontaneously growing transverse waves in a plasma due to an anisotropic velocity distribution, Phys. Rev. Lett., 2, 83-84, 1959.

Zaheer, S. and Murtaza, G.:, Weibel instability with nonMaxwellian distribution functions, Phys. Plasmas, 14, 022108, doi:10.1063/1.2536159, 2007. 